\title{
TRAUMATISMO DENTÁRIO: QUAL O CONHECIMENTO DO CIRURGIÃO DENTISTA?
}

\author{
Mariana Esperendi Bastianini, Andressa Restani Oliveira, Graziela Garrido Mori Panucci, Carolina dos Santos \\ Santinoni, Juliane Avansini Marsicano, Rosana Leal do Prado
}

Universidade do Oeste Paulista - UNOESTE, Presidente Prudente, SP. e-mail: marianaesperendi@gmail.com

\section{RESUMO}

Os objetivos deste estudo foram avaliar o conhecimento dos cirurgiões-dentistas sobre ao diagnóstico e tratamento de situações clínicas de traumatismo dentário e o impacto de uma atividade de orientação no formato de palestra no processo formativo dos profissionais. O instrumento de avaliação foi um questionário que continha informações profissionais e diferentes casos clínicos de traumatismo dentário para que os cirurgiões dentistas respondessem. Os dados foram coletados em duas etapas, sendo antes da realização da palestra e após a mesma. Para análise estatística foi empregado o teste $G$, com nível de significância de $5 \%$. A maioria dos profissionais eram clínicos gerais $(71,4 \%)$, sendo que a maior parte já havia tido experiência com traumatismo dentário (75,5\%). No entanto, quando realizada a análise estatística, apenas a conduta perante dente com exposição pulpar $>2 \mathrm{~mm}$ com rizogênese incompleta e conduta após reimplante imediato de dente avulsionado com rizogênese completa, demonstraram resultados positivos com diferenças estatisticamente significantes. Visto isso, concluímos que os profissionais possuíam conhecimento satisfatório, com ressalvas para casos mais complexos. No entanto, atividade de orientação, não contribuiu para a melhora nas decisões clinicas.

Palavras-chave: traumatismos dentários, avulsão dentária, conhecimento, odontólogos, promoção da saúde.

\section{DENTAL TRAUMATISM: WHAT IS THE KNOWLEDGE OF THE DENTIST SURGEON?}

\begin{abstract}
The aims of this study were to evaluate the knowledge of dentists on the diagnosis and treatment of clinical situations of dental trauma and the impact of an orientation activity in the form of a lecture on the training process of professionals. The assessment instrument was a questionnaire that contained professional information and different clinical cases of dental trauma for dentists to answer. Data were collected in two stages, before and after the lecture. For statistical analysis, the $\mathrm{G}$ test was used, with a $5 \%$ significance level. Most professionals were general practitioners (71.4\%), and most of them had experience with dental trauma (75.5\%). However, when the statistical analysis was carried out, only the conduct before a tooth with pulp exposure $>2 \mathrm{~mm}$ with incomplete rhizogenesis and management after immediate re-implantation of avulsed tooth with complete rhizogenesis, demonstrated positive results with statistically significant differences. In view of this, we concluded that the professionals had satisfactory knowledge, with reservations for more complex cases. However, orientation activity did not contribute to the improvement in clinical decisions.
\end{abstract}

Keywords: tooth injuries, tooth avulsion, knowledge, dentists, health promotion.

\section{INTRODUÇÃO}

O traumatismo dentário corresponde a uma injúria que pode acometer tanto o dente quanto os tecidos que o suportam. Eles são consequências de uma transmissão aguda de energia a essas estruturas, que dentre outras situações podem ocorrer principalmente devido a quedas, acidentes automobilísticos, violência e acidentes durante atividades esportivas ${ }^{1-4}$. 
Os danos causados pelos traumas bucais resultam em consequências que transcendem os problemas estéticos, prejudicando o bem-estar funcional, psicológico e social, além de gerar custos para as vítimas. Tal fato o torna um dos principais agravos em saúde bucal, bem como um significante problema de saúde pública ${ }^{1,5-9}$.

A incidência do trauma é maior em crianças e adolescentes ${ }^{5,10}$, na dentição permanente ${ }^{1,11,12}$ e mais frequentemente nos incisivos centrais superiores ${ }^{1,7,10,11,13,14}$, fato justificado pela existência de alguns fatores predisponentes como o overjet acentuado e o vedamento labial deficiente, que expõem os incisivos maxilares ${ }^{10,11}$.

Os traumatismos dentários são divididos em: fratura dentária e de osso alveolar, luxações e avulsão ${ }^{15,16}$. Dentre estes, a avulsão é considerada a situação de maior severidade, uma vez que o elemento dentário se desloca completamente do interior do alvéolo. A reimplantação, na maioria dos casos, é o tratamento indicado, exceto na presença de lesão cariosa extensa, doença periodontal intensa e condições sistêmicas que exigem $o$ uso de medicações severas ${ }^{16}$. Para 0 sucesso do reimplante é importante que o elemento avulsionado tenha o menor tempo possível fora do alvéolo e um armazenamento adequado (leite, saliva, soro fisiológico ou água), assim existirá uma maior possibilidade de sobrevivência das células periodontais e consequentemente, uma melhor reinserção das fibras do ligamento periodontal ${ }^{17}$.

Algumas medidas se tornam necessárias para prevenção dos traumatismos dentários, dentre elas a intervenção ortodôntica nos casos de overjet acentuado, que deve ser realizada o mais breve possível ${ }^{7}$. Além disso, estudos evidenciam a importância do desenvolvimento de atividades que orientem sobre os primeiros socorros frente a casos de traumas $^{18}$, principalmente a professores, educadores físicos, bombeiros, esportistas e cirurgiões-dentistas, uma vez que são os indivíduos que estão em maior contato com casos de traumatismos ${ }^{19}$.

O prognóstico de sucesso dos tratamentos de dentes traumatizados depende do atendimento emergencial oferecido pelo cirurgião-dentista ${ }^{13}$, uma vez que a negligência ou imperícia pode acarretar em inúmeras sequelas como: reabsorções internas e externas, calcificações pulpares, necrose pulpar, anquilose dentária e até a perda do dente. Portanto, o cirurgião-dentista deve ter o conhecimento necessário para fazer o manejo desses casos, sabendo diagnosticar, planejar e realizar o tratamento correto e acompanhar por período adequado, de acordo com a condição clínica ${ }^{5,13}$.

Dado o antagonismo presente na literatura sobre o conhecimento dos profissionais da odontologia, é de suma importância avaliar o conhecimento dos cirurgiões dentistas sobre o manejo de casos de traumatismos dentários e o quão eficaz é a atividade de orientação, uma vez que tem sido uma das modalidades mais utilizadas para orientação profissional.

Este estudo teve o objetivo avaliar o conhecimento dos cirurgiões-dentistas sobre ao diagnóstico e tratamento de situações clínicas de traumatismo dentário. Adicionalmente, avaliou o impacto de uma atividade de orientação no formato de palestra no processo formativo dos profissionais.

\section{METODOLOGIA}

Trata-se de um estudo longitudinal e descritivo. As coletas foram realizadas com cirurgiões-dentistas de uma cidade de médio porte do interior de São Paulo. A pesquisa iniciou-se após a aprovação do Comitê de Ética em Pesquisa, sendo o CAAE 90394918.2.0000.5515.

Os dentistas foram orientados sobre os objetivos desta pesquisa e só pôde participar após a assinatura do Termo de Consentimento Livre e Esclarecido. Este termo inclui a descrição de como o participante será analisado, garantindo o sigilo da informação $e$ desautorizando qualquer forma de coação ou pressão pela sua participação voluntária.

A amostra foi obtida por conveniência e foram convidados a participar da pesquisa todos os 96 cirurgiões-dentistas, devidamente inscritos no Conselho Regional de Odontologia de São Paulo e que atuam no Sistema Único de Saúde do município em questão. A abordagem foi realizada coletivamente, durante uma atividade ordinária que é oferecida pela Secretaria Municipal de Saúde a estes profissionais.

A avaliação foi realizada em duas etapas, ambas através de um mesmo questionário ${ }^{7}$, que era dividido em duas partes. A parte I envolvia as informações pessoais e profissionais dos participantes, como: gênero, idade, local de formação, especialidade, se tinha alguma experiência com traumatismo e se já havia feito algum curso para o manejo do traumatismo. Já a 
parte II, continha situações clínicas para o profissional solucionar, conforme exposto no Quadro 1, sendo que foram disponibilizadas de 4 a 5 alternativas para a resposta, sendo que uma delas incluía "não sei".

Quadro 1. Situações que compõem o questionário, baseado no estudo de Hu et al. ${ }^{7}$.

\begin{tabular}{|c|l|}
\hline Caso clínico 1 & $\begin{array}{l}\text { Uma criança de 7 anos chegou ao consultório com uma fratura de coroa complicada em um } \\
\text { dente anterior imaturo e apresentava exposição pulpar menor que 1 mm e tempo decorrido } \\
\text { desde o trauma menor que uma hora. Qual procedimento é indicado para ser realizado? }\end{array}$ \\
\hline Caso clínico 2 & $\begin{array}{l}\text { Uma criança de 7 anos chegou ao consultório com uma fratura de coroa complicada em um } \\
\text { dente anterior imaturo e apresentava exposição pulpar maior que } 2 \mathrm{~mm} \text { e tempo decorrido } \\
\text { desde o trauma maior que uma hora. Qual procedimento é indicado para ser realizado? }\end{array}$ \\
\hline Caso clínico 3 & $\begin{array}{l}\text { A mobilidade de um dente é evidente. O Raio-X revela fratura no terço médio da raiz do } \\
\text { incisivo central superior maduro. A injúria aconteceu há 1 dia. Qual procedimento é indicado } \\
\text { para ser realizado? }\end{array}$ \\
\hline Caso clínico 4 & $\begin{array}{l}\text { Quais são os resultados esperados e as modalidades de tratamento usadas para uma fratura } \\
\text { radicular no terço médico ou apical dentário? }\end{array}$ \\
\hline Caso clínico 5 & $\begin{array}{l}\text { A intrusão de um incisivo central imaturo (de aproximadamente 5 mm) aconteceu 2h atrás. } \\
\text { Qual procedimento é indicado para ser realizado? }\end{array}$ \\
\hline Caso clínico 6 & $\begin{array}{l}\text { Uma luxação lateral de um dente anterior ocorreu há 1h e o dente não responde aos testes } \\
\text { térmicos. O raio-X revela destruição óssea periapical. Isso está relacionado com: }\end{array}$ \\
\hline Caso clínico 7 & A reabsorção de substituição está associada a: \\
\hline Caso clínico 8 & $\begin{array}{l}\text { As condições para o melhor prognóstico de um dente avulsionado em seco são quando o } \\
\text { reimplante acontece: }\end{array}$ \\
\hline Caso clínico 9 & $\begin{array}{l}\text { Uma mãe ligou para o consultório dental explicando que sua filha tinha acabado de } \\
\text { "nocautear" um dente permanente anterior. Sua recomendação é: }\end{array}$ \\
\hline Caso clínico 10 & $\begin{array}{l}\text { Uma criança chegou ao consultório odontológico com avulsão dentária de um elemento com } \\
\text { rizogênese incompleta que já havia sido reimplantado dentro de 30 min após o trauma (no } \\
\text { local do acidente). Você executará: }\end{array}$ \\
\hline Caso clínico 11 & $\begin{array}{l}\text { Um dente avulsionado com rizogênese completa foi reimplantado dentro de 30 min após o } \\
\text { trauma ocorrer. Você executará: }\end{array}$ \\
\hline
\end{tabular}

Os dados da primeira etapa foram coletados e em seguida os voluntários foram convidados a participar de uma atividade de orientação realizada num projeto de extensão da Universidade do Oeste Paulista. A atividade contou com o uso de banners e palestras.

Os participantes foram orientados sobre o reimplante imediato, sobre o modo apropriado de lavagem do dente avulsionado, bem como os meios de conservação para este dente e condutas pós-traumatismo. Também, foram encorajados a discutirem suas dúvidas e colaborarem com o conhecimento e aprendizado de todos.

Após 6 meses da realização da atividade, os participantes foram reavaliados com a aplicação do mesmo instrumento, para avaliar o impacto do projeto de extensão sobre o conhecimento do profissional frente ao assunto proposto.
Para análise estatística foi empregado o teste $\mathrm{G}$, considerando o nível de significância de $5 \%$.

\section{RESULTADOS}

Na primeira avaliação apenas 49 (51,04\%) profissionais aceitaram participar desta pesquisa, tendo esse número reduzido para 20 (20,83\%) profissionais na segunda avaliação. Em ambos os momentos 0 número de mulheres foi predominante. A maioria dos profissionais era de Clínicos Gerais, sendo que a maior parte já havia tido experiência com traumatismo dentário, no entanto, na primeira avaliação $63,24 \%$ disseram nunca ter feito algum curso sobre o assunto, diferente do que aconteceu na segunda avaliação, na qual esse número reduziu para $25 \%$ (Tabela 1). 
Tabela 1. Características pessoais e profissionais dos cirurgiões dentistas voluntários.

\begin{tabular}{lccc}
\hline Variáveis & & Primeira Avaliação & Após 6 meses \\
\hline Gênero (\%) & Masculino & 22,45 & 25 \\
Média de Idade & Feminino & 77,55 & 75 \\
(anos) & Homens & 44,54 & 41,6 \\
Universidade de formação & Mulheres & 48,60 & 45,2 \\
(\%) & UNOESTE & 67,34 & 60 \\
& USP & 12,24 & 15 \\
& UNESP & 2,04 & 5 \\
Média de tempo de & Outras & 18,36 & 20 \\
formação (anos) & Homens & 20,20 & 21 \\
& Mulheres & 26,13 & 22,13 \\
Especialidade (\%) & Clínico geral & 71,43 & 70 \\
& Endodontista & 2,04 & 5 \\
Experiência com & Outros & 26,53 & 15 \\
traumatismo dentário (\%) & Sim & 75,50 & 65 \\
Realização de curso de & Não & 24,50 & 35 \\
capacitação para & Sim & 36,76 & 75 \\
traumatismo dentário (\%) & Não & 63,24 & 25 \\
\hline
\end{tabular}

As duas primeiras perguntas da segunda etapa tratavam de exposição pulpar menor que 1 $\mathrm{mm}$ e maior que $2 \mathrm{~mm}$, respectivamente. A quantidade de acertos aumentou na segunda avaliação em ambas as questões, no entanto, só foi encontrada diferença estatística significante na segunda pergunta (Tabela 2), na qual os acertos foram $100 \%$.

Tabela 2. Quantidade de acertos das perguntas relacionadas ao tratamento em casos de exposição pulpar.

\begin{tabular}{lccc}
\hline Pergunta & Antes (\%) & Depois (\%) & $\mathbf{p}$ \\
\hline $\begin{array}{l}\text { Dente com exposição pulpar }<1 \mathrm{~mm} \\
\text { com rizogênese incompleta }\end{array}$ & 83,30 & 88,90 & $>0,05$ \\
$\begin{array}{l}\text { Dente com exposição pulpar }>2 \mathrm{~mm} \\
\text { rizogênese incompleta }\end{array}$ & 74,35 & 100 & $=0,05$ \\
\hline
\end{tabular}

Na terceira e quarta situação, também houve o aumento do número de acertos, porém, nenhuma diferença estatística foi encontrada (Tabela 3). Diferente do que se observou nas perguntas 5, 6 e 7, que apresentou uma redução do número de acertos, mas da mesma forma, nenhuma diferença estatística significante foi observada (Tabela 4). 
Tabela 3. Quantidade de acertos das perguntas sobre manejo nos casos de fraturas.

\begin{tabular}{lccc}
\hline Pergunta & Antes (\%) & Depois (\%) & p \\
\hline $\begin{array}{l}\text { Fratura do terço médio } \\
\text { radicular }\end{array}$ & 61,22 & 65 & $>0,05$ \\
$\begin{array}{l}\text { Resultados esperados de } \\
\text { fraturas terços médio e apical }\end{array}$ & 24,48 & 25 & $>0,05$ \\
\hline
\end{tabular}

Tabela 4. Quantidade de acertos das perguntas relacionadas aos casos de luxação e reabsorção radicular.

\begin{tabular}{lccc}
\hline Pergunta & Antes (\%) & Depois (\%) & p \\
\hline Tratamento de luxação intrusiva $(5 \mathrm{~mm})$ & 81,63 & 70 & $>0,05$ \\
de dente com rizogênese incomplenta & 6,12 & 5 & $>0,05$ \\
Consequência da luxação lateral & 16,32 & 15 & $>0,05$ \\
Reabsorção radicular por substituição &
\end{tabular}

Pra finalizar, as perguntas $8,9,10$ e 11 , tratavam das condutas frente à diferentes situações nos casos de avulsão dentária. Foi encontrada diferença estatisticamente significante na pergunta 8 , que envolvia 0 prognóstico do dente avulsionado e o número de acerto foi menor na segunda avaliação, assim como, na pergunta 11 , sendo que nessas os valores foram positivas, tendo melhorado o conhecimento dos profissionais sobre a conduta após reimplante imediato de dente avulsionado com rizogênese completa. Para as questões 9 e 10, nenhuma diferença estatística foi observada (Tabela 5).

Tabela 5. Quantidade de acertos das perguntas relacionadas ao manejo dos casos de avulsão dentária.

\begin{tabular}{lccc}
\hline Pergunta & Antes (\%) & Depois (\%) & p \\
\hline $\begin{array}{l}\text { Determinação do melhor prognóstico para o } \\
\text { dente avulsionado }\end{array}$ & 91,83 & 70 & $=0,03$ \\
$\begin{array}{l}\text { Orientação para paciente que acabou de } \\
\text { sofrer avulsão }\end{array}$ & 95,91 & 90 & $>0,05$ \\
$\begin{array}{l}\text { Conduta após reimplante imediato de dente } \\
\text { avulsionado com rizogênese incompleta }\end{array}$ & 83,67 & 95 & $>0,05$ \\
$\begin{array}{l}\text { Conduta após reimplante imediato de dente } \\
\text { avulsionado com rizogênese completa }\end{array}$ & 36,73 & 70 & $=0,02$ \\
\hline
\end{tabular}

\section{DISCUSSÃO}

Os traumas dentários são muito comuns na população, principalmente em crianças e adolescentes $^{21}$. Eles afetam consideravelmente a qualidade de vida dos indivíduos, trazendo alterações bucais que geram desconforto físico e emocional $^{5-10}$. Visto isso, a decisão e conduta clinica do cirurgião dentista durante o tratamento emergencial do paciente é determinante para propiciar um melhor prognóstico para o dente traumatizado.

Neste estudo, de $65 \%$ a $75,5 \%$ dos profissionais entrevistados já tiveram alguma experiência com traumatismo dentário. Não há uma unanimidade nos estudos que pesquisam o conhecimento dos cirurgiões dentistas, sobre o manejo de traumas dentários. Parte deles demonstraram que os profissionais têm o conhecimento necessário, mas apresentam algumas limitações para casos mais complexos $^{2,5,13,14}$. Verificou-se também que especialistas possuem conhecimento mais apurado $^{1}$, contrapondo outros estudos que mostraram que o conhecimento sobre o assunto é moderado ${ }^{12,20}$ ou baixo ${ }^{7,17}$. 
Os resultados deste estudo mostram que o nível de conhecimento dos profissionais é parcialmente satisfatório, sendo que as maiores dificuldades e menores números de acertos aconteceram nas perguntas de maior complexidade, que exigiam um conhecimento específico. Pode-se atribuir a isso o fato de que a maioria dos cirurgiões dentistas entrevistados eram clínico gerais $(70 \%-71,43 \%)$ e desse modo, quando o manejo exigia um conhecimento endodôntico específico, surgiam as dificuldades em responder às situações clínicas apresentadas no questionário. Tal fato, corrobora com o estudo de $\mathrm{Hu}$ et al. ${ }^{7}$, o qual foi encontrado que os endodontistas demonstraram maiores acertos comparados com os clínicos gerais. Outra hipótese é de que os profissionais endodontistas acabam tendo mais contato com os casos de traumas dentários, diminuindo dessa forma, a experiência dos clínicos gerais ${ }^{23}$, o que os impedem de buscarem maiores conhecimentos sobre traumatismo dentário.

As perguntas relacionadas à modalidades de tratamento de fraturas radiculares no terço médio e apical, consequências da luxação lateral, característica da reabsorção radicular por substituição e a conduta após reimplante imediato de dente avulsionado com rizogênese completa, foram as que obtiveram menores porcentagens de acertos (Tabela 3, 4 e 5). Estas questões exigem um conhecimento que vai além da prática realizada pelo cirurgião dentista, envolvendo um conhecimento teórico que é necessário para a conduta desses casos. Os dados obtidos dos questionários, quando comparados após a atividade de orientação, mostrou maior número de acertos com relação a conduta após reimplante imediato de dente avulsionado com rizogênese completa, com diferença estatisticamente significante neste caso isolado relacionado à avulsão dentária (Tabela 5).

Visto a quantidade de casos para serem solucionados pelos profissionais, apenas o caso clínico 2 obteve, ao final, 100\% de acertos, com diferença estatística significante (Tabela 2). Dessa forma, acredita-se que a atividade de orientação no formato de palestra, não seja a melhor maneira de abordar o traumatismo dentário com os profissionais. Diferente do que se observou no estudo com professores de educação infantil de Mori et al. ${ }^{18}$, que apresentou efetividade na atividade de orientação, melhorando 0 conhecimento desses profissionais.
Alguns fatores podem ter influenciado na dificuldade dos cirurgiões dentistas em adquirir conhecimento durante a palestra, como por exemplo, a presença de muitos profissionais pautada somente nos benefícios que recebem por participarem de palestras oferecidas por seu empregador, não se importando com o conteúdo oferecido e não dando a devida atenção à explicação dada; a falta de experiência com casos de traumatismos dentários, por às vezes não ser a realidade da população que ele atende, diminuindo o seu interesse pelo assunto abordado $^{23} ;$ e até mesmo o formato de abordagem do assunto, que pode ter trazido alguma confusão durante as explicações, dificultando o aprendizado dos indivíduos. Stangler et al. ${ }^{24}$, observaram que após 4 meses da realização de palestras houve a perda de conhecimento pelos indivíduos, demonstrando mais uma dificuldade desse tipo de abordagem.

Concluímos que os cirurgiões-dentistas possuem um conhecimento satisfatório sobre traumatismo dentário em situações cínicas de baixa e média complexidade, com ressalvas para casos mais complexos, que não exigem conhecimento específico. No entanto, após a participação dos voluntários da atividade de orientação, não houve melhora nas decisões clinicas.

\section{AGRADECIMENTOS}

Este estudo teve o apoio da Coordenação de Aperfeiçoamento de Pessoal de Nível Superior (CAPES).

\section{CONFLITO DE INTERESSE}

Os autores declaram não haver qualquer potencial conflito de interesse que possa interferir na imparcialidade deste trabalho científico.

\section{REFERÊNCIAS}

1. Pinheiro SAA, Delfino CS. Conhecimento do cirurgião-dentista sobre trauma dentário. Rev Cir Traumatol Buco-Maxilo-Fac. 2014;14(1):83-90.

2. Re D, Augusti D, Paglia G, Augusti G, Cotti E. Treatment of traumatic dental injuries: evaluation of knowledge among Italian dentists. Eur J Paediatr Dent. 2014;15(1).

3. Barbosa CL, Lacerda RA, Alves AC. Análise do nível de conhecimento dos odontopediatras sobre prevenção de traumatismos relacionados a 
esportes. J Bras Odontopediatr Odontol Bebê. 2003;6(33):399-404.

4. Vasconcellos LGO, Brentel AS, Vanderlei AD, Vasconcellos LMR, Valera MC, Araújo MAM. Knowledge of general dentists in the current guidelines for emergency treatment of avulsed teeth and dental trauma prevention. Dent Traumatol. 2009;25:578-83. DOI: https://doi.org/10.1111/j.1600$\underline{\text { 9657.2009.00820.x }}$

5. Granville-Garcia AF, Menezes VA, Lopes I, Araujo PS, Fontes LBC, Cavalcanti AL. Conduta terapêutica dos cirurgiões-dentistas em relação aos traumatismos dentários. Arq Ciênc Saúde Unipar. 2008;12(3):239-47.

6. Zhao Y, Gong Y. Knowledge of emergency management of avulsed teeth: a survey of dentists in Beijing, China. Dent Traumatol. 2010;26:281-4.

DOI:

https://doi.org/10.1111/j.1600-

$\underline{9657.2010 .00877 . x}$

7. Hu LW, Prisco CRD, Bombana AC. Knowledge of Brazilian general dentists and endodontists about the emergency management of dento-alveolar trauma. Dent Traumatol. 2006;22:113-7. DOI: https://doi.org/10.1111/i.1600-

$\underline{\text { 9657.2006.00341.x }}$

8. Ramos-Jorge ML, Bosco VL, Peres MA, Nunes ACGP. The impact of treatment of dental trauma on the quality of life of adolescentes - a casecontrol study in southern Brazil. Dent Traumatol. 2007;23:114-9.

DOI: https://doi.org/10.1111/i.16009657.2005.00409.x

9. Antunes LAA, Rodrigues AS, Martins AMC, Cardoso ES, Homsi N, Antunes LS. Traumatic dental injury in permanente teeth: knowledge and management in a group of Brazilian school teachers. Dent Traumatol. 2015. DOI: https://doi.org/10.1111/edt.12249

10. Cohenca N, Forrest JL, Rotstein I. Knowledge of oral health professional of treatment of avulsed teeth. Dent Traumatol. 2006;22:296-301. DOI: $\quad$ https://doi.org/10.1111/i.1600$\underline{\text { 9657.2005.00448.x }}$
11. Zaleckiene V, Peciuliene V, Brukiene V, Drukteinis S. Traumatic dental injuries: etiology, prevalence and possible outcomes. Baltic Dental Maxillofacial J. 2014;16(1):7-14.

12. Akhlaghi N, Nourbakhsh N, Khademi A, Karimi L. General dental practioners knowledge about the emergency management of dental trauma. Iran Endod J. 2014;9(4):251-6.

13. Abu-Dawoud M, Al-Enezi B, Andersson L. Knowledge of emergency management of avulsed teeth among young physicians and dentists. Dent Traumatol. 2007;23:348-55. DOI: https://doi.org/10.1111/j.1600-

$\underline{9657.2006 .00477 . x}$

14. Skaare AB, Pawlowski AA, Aas ALM, Espelid I. Dentists self-estimation of their competence to treat avulsion and root fracture injuries. Dent Traumatol. 2015;31:368-73. DOI: https://doi.org/10.1111/edt.12186

15. DiAngelis AJ, Andreasen JO, Ebeleseder KA, Kenny DJ, Trope $M$, Sigurdsson $A$ et al. International Association of Dental Traumatology guidelines for the management of traumatic dental injuries: 1. Fractures and luxations of permanent teeth. Dent Traumatol. 2012;28:2-12. DOI: $\quad$ https://doi.org/10.1111/i.16009657.2011.01103.x

16. Andersson L, Andreasen JO, Day P, Heithersay $G$, Trope $M$, DiAngelis AJ et al. International Association of Dental Traumatology guidelines for the management of traumatic dental injuries: 2 . Avulsion of permanent teeth. Dent Traumatol. 2012;28:88-96. DOI: https://doi.org/10.1111/j.16009657.2012.01125.x

17. Santos MESM, Neto MGG, Souza CMA, Soares DM, Plameira PTSS. Nível de conhecimento dos profissionais de Enfermagem, Educação Física e Odontologia sobre traumatismo dentoalveolar do tipo avulsão. Rev Cir Traumatol Buco-Maxilo-fac. 2010;10(1):95-102.

18. Mori GG, Castilho LR, Nunes DC, Turcio KHL, Molina RO. Avulsion of permanent teeth: analysis of the efficacy of an informative campaign for professionals from elementary schools. J Appl Oral Sci. 2007;15(6):534-8. DOI: 
http://dx.doi.org/10.1590/S1678-

$\underline{77572007000600015}$

19. Santos CAO, Caldas ATL, Souza RLF, Siewes M, Carvalho MMP, Campos FAT. Dental traumatisms: primary health care dentists' knowledge. Rev Família, Ciclos Vida Saúde Contexto Social. 2018:6(2).

DOI: https://doi.org/10.18554/refacs.v6i2.2812

20. Aijazairy $\mathrm{YH}$, Halawany $\mathrm{HS}$, Almaflehi $\mathrm{N}$, Alhussainan NS, Abraham NB, Jacob V. Knowledge about permanent tooth avulsion and its management among dentists in Riyadh, Saudi Arabia. BMC Oral Health 2015;15:135. DOI: https://doi.org/10.1186/s12903-015-0126-3

21. Andersson L. Epidemiology of traumatic dental injuries. JOE 2013 Mar;39(3s). DOI: https://doi.org/10.1016/i.joen.2012.11.021

22. França RI, Traebert J, De Lacerda J T. Brazilian dentists' knowledge regarding immediate treatment of traumatic dental injuries. Dent Traumatol. 2007;23(5): 287-90. DOI: https://doi.org/10.1111/j.1600-

9657.2006.00444.x

23. Jackson NG, Waterhouse PJ, Maguire A. Management of dental trauma in primary care: a postal survey of general dental practitioners. Brit Dent Journal. 2005;198(5):293-7.

24. Stangler ML, Echer R, Vanni JR. Avaliação quantitativa do conhecimento dos estagiários do curso de Pedagogia - UPF sobre avulsãoreimplante. RFO UPF. 2002;7(1):23-28. 